\title{
Treatment compliance in schizophrenia
}

\author{
Alec Buchanan
}

There is a myth that people with mental disorders comply poorly with treatment. In fact, psychiatric patients are no more likely than patients in other medical specialities to go against the advice of their doctor. However, it is easy to find instances where psychotropic medication is refused by the supposed beneficiary. The value of neuroleptic treatment in schizophrenia is now widely accepted, and failure to take such treatment is associated with relapse. Relapse may endanger the patient and other people. Despite this, people with schizophrenia frequently fail to take their treatment. One-third of patients leaving a general adult psychiatry ward can be expected to be non-compliant within two years (Buchanan, 1992).

\section{When do patients fail to take their treatment?}

The factors linked to compliance can be divided into a number of areas as shown in Box 1 .

\section{Socio-demographic characteristics}

Socio-demographic variables are not important predictors of compliance. There are some suggestions, from research in general medicine, that old people comply better (Baekeland \& Lundwall, 1975). There is some evidence also that low socio-economic status and unemployment are associated with poor compliance. Little work has been done on exclusively psychiatric populations.

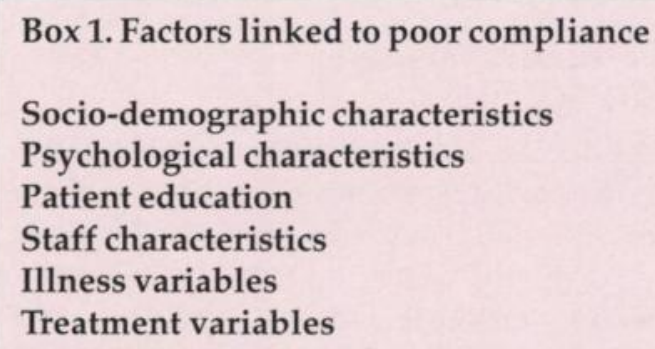

\section{Psychological characteristics}

A diagnosis of sociopathic personality as well as 'impulsivity' and a disregard for rules and regulations have been linked to poor compliance, a conclusion which seems somewhat circular. When more specific psychological characteristics have been studied, denial of illness and lack of insight have shown the same association. Detailed studies of patient attitudes to illness and treatment (without recording levels of drug compliance) have shown that 'illness positive' attitudes such as: "in a way, my illness helped me grow up" are associated with a lower rate of re-hospitalisation (Soskis \& Bowers, 1969). The importance of such research was emphasised by Hogan et al (1983), who tested a self-report scale in a group of patients with schizophrenia. The authors were able to consign $89 \%$ of their sample to compliant and non-compliant groups using a 30 -point scale which asked patients how they felt on medication without reference to their levels of knowledge. 


\section{Patient education}

Patient education to improve levels of knowledge has been ascribed a crucial role in maintaining treatment adherence in patients with schizophrenia. Much of the research is methodologically weak and the conclusion that levels of education affect compliance has been challenged (for review see Ley \& Morris, 1984). The explanation for the contradictory nature of much of the evidence may lie in the factors which inevitably accompany intensive patient education, factors such as increased contact with interested and motivated health professionals.

\section{Staff characteristics}

Different doctors achieve different levels of compliance. The reasons are not clear. Doctor characteristics associated with improved compliance include: an 'ability to inspire trust', an 'accepting' manner and a 'task-oriented' and 'flexible' attitude. It has been shown, by studies which directly observe interview technique, that those with low drop-out rates conduct more personalised interviews with a clearer structure and focus. The interaction between doctor and patient has not been widely studied. Where research does exist the substantial methodological problems involved make the drawing of conclusions difficult. It seems that disagreements over medication do not significantly impair attendance, and an atmosphere of negotiation is said to improve matters. There is general agreement that improved supervision is associated with improved compliance.

\section{Illness variables}

The severity of someone's illness, even when it is reflected in the level of psychopathology when they are discharged, is probably not related to compliance. Persecutory delusions are frequently mentioned as placing a patient at risk of complying poorly but the research findings in this regard are inconsistent. Only grandiosity, perhaps linked to a sense of well-being when ill, has shown a consistent link.

\section{Treatment variables}

Treatment variables which have been related to compliance can be reduced to three categories. The complexity of the drug regimen has been found to show an inverse relationship with compliance, reflecting the difficulty which many patients experience in understanding their doctors' instructions. Probably both dose frequency and the total number of drugs prescribed are important. The relative value of oral and depot medication has been investigated specifically with regard to schizophrenia. The usual conclusion is that depot preparations are better complied with than oral medication. Side-effects fail to show the consistent relationship with poor compliance which might be expected, perhaps because studies often fail to take into account their severity. Of those which have been implicated, akathisia and akinesia are most often blamed.

\section{Measures which improve compliance in randomised trials}

Many papers have been written about how to improve compliance. Less common are controlled trials which randomise cases between treatment and control groups. Didactic educational methods have been shown to be ineffective in three of these (Streicker et al, 1986; Brown et al, 1987; MacPherson et al, 1996). Three techniques have been shown to work.

A team led by Kemp and David at the Institute of Psychiatry has developed an approach which they call compliance therapy (Kemp et al, 1996). The intervention borrows heavily from Miller \& Rollnick's (1991) 'motivational interviewing', originally developed for use in drug rehabilitation. The therapist focuses on the value of staying well, the need for prophylactic treatment and uses metaphors such as using drugs as a 'protective layer' and as an 'insurance policy'. It consists of six sessions, each of which lasts between 20 and 60 minutes. The first two are used to review the patient's history of illness. During the next two symptoms and side-effects are discussed, as well as any ambivalence which the patient describes towards treatment. In the last two sessions the therapist addresses the stigma of drug treatment and emphasises the value of staying well.

Forty-seven patients were randomly assigned to treatment and control groups. The 47 were inpatients on an acute general psychiatry ward. All of them suffered from psychoses and, as a group, they were more unwell than most research populations. Their mean score on the Brief Psychiatric Rating Scale (BPRS; Overall \& Gorham, 1962) was 60, over 
half of the group were detained under the Mental Health Act 1983 and they received, on average, the equivalent of $850 \mathrm{mg}$ chlorpromazine per day. Instead of compliance therapy, the control group received supportive counselling where the therapist spent his or her time listening to the patients' concerns. Compliance was rated on a seven-point scale which ranged from complete refusal, which scored one, to active participation in treatment, which scored seven. The result was an improvement in the treatment group's compliance score, from an average of 3.4 before treatment to an average of 5.6 six months after treatment. The score before treatment indicates accepting treatment reluctantly and questioning its value. The score six months after treatment indicates that the patient takes more interest in, and responsibility for, the course of treatment being prescribed.

The strengths of the study are that the setting was one which most clinicians would recognise and that the patients were similar to those one might meet on an acute admission ward in any general psychiatric hospital. One weakness was the number of dropouts. Thirty-one per cent of those considered eligible refused to participate and a further eight, $17 \%$ of the study group, dropped out in the course of the research. Another is that the group as a whole was relatively compliant. Even before any treatment, the mean compliance score was one which corresponded to a patient taking his or her treatment. After treatment the subjects were more enthusiastic about their treatment, but the problem which faces general psychiatrists is not that some patients take their treatment reluctantly, it is that some patients do not take their treatment at all. It could be argued that the really non-compliant patients were those who dropped out of the study.

Eckman et al (1992) also conducted a randomised controlled trial, this time of 401 male patients of the Veteran's Administration in the USA. Their subjects were a mixture of in-patients and out-patients and were receiving far less medication: between 5 and $10 \mathrm{mg}$ fluphenazine every two weeks. The treatment consisted of 'modular skills training'. For six months, twice weekly, the subjects were taught to identify the warning signs of relapse, how to cope with symptoms, how to take drugs properly and what the side-effects of those drugs were likely to be. The rationale offered by the authors was that the symptoms of schizophrenia both distract patients from taking in information or, in the case of what they see as sustained deficits in attention, memory and executive functioning, make it difficult for them to retain and use information even if they are not distracted. These handicaps can be overcome, the authors argue, by providing information in a structured and repetitive way. The control treatment was group psychotherapy and the follow-up period was 12 months.

Thirty-four per cent of the subjects dropped out. Outcome was measured in terms of each subject's performance in seven role-playing tests, which addressed each of the areas of knowledge which had been covered by the modular skills training. Compliance itself was not measured. The authors found that those subjects who had received the training did substantially better on the tests, and that this improvement was maintained over the 12 months of follow-up. There was no relationship between a subject's ability to benefit from the training and their level of psychopathology, as measured by the BPRS, at the start of the training. This might appear to go against the authors' rationale for their treatment, that the symptoms of schizophrenia impair learning. The authors argue, however, that it shows that modular skills training is sufficiently powerful to overcome this effect.

Boczkowski et al (1985) recruited 36 male patients with schizophrenia from the Veterans' Administration Hospital out-patient clinic in Augusta in the USA. They were randomly assigned to three groups of 12 to receive education, 'behavioural tailoring' or a control treatment. The 12 in the behavioural tailoring group spent between 30 and 50 minutes discussing, with a therapist, ways of tailoring their drug regime to fit in with their daily routine. They were given a calendar with a dated slip of paper corresponding to each dose of neuroleptic but little information as to why they should take it. Those in the educational group spent the same length of time being given information about their condition, while those in the control group discussed what they thought of the hospital and their time in the army. Compliance was measured by pill count and follow-up was for three months. The behavioural tailoring group was significantly more compliant at three months, taking, on average, $85 \%$ of their medication compared with $70 \%$ in the control group. The educational package had no effect.

The strength of the study is that none of the 36 participants dropped out, and that the intervention could easily be incorporated into most out-patient treatment settings. The drawbacks, apart from the small numbers and the short follow-up period, concern the nature of the sample. The subjects were drawn from an out-patient population all of whom had been taking their medication, voluntarily and regularly, for two years. Before entering the study they were being seen, on average, monthly by their psychiatrists and had last been inpatients, on average, five years earlier. This suggests that they were relatively well and wellbehaved, at least in terms of their drug taking. The 
applicability to other groups of patients is yet to be demonstrated.

\section{Improving the compliance of people with schizophrenia}

Patients are not 'passive receptacles for treatment'.

(Corrigan et al, 1990)

A theme emphasised by many reviews is that doctors should aim to establish and maintain a joint approach to treatment with the patient (see Box 2). Listening to the patient, empathising, providing comprehensible information and negotiating treatment contracts are the means of attaining this goal, and the means seem no more exceptionable than the ends. One practical suggestion is to present medication as something which increases the person's control over their own life and gives them options which they would not have without it. Knowing what a patient enjoys doing allows a psychiatrist to explain how taking medication may make that patient better able to do it. Knowledge of the detail of people's lives allows a doctor or nurse to discuss, rather than simply refute or confirm, statements like: "since I stopped taking medication I feel exactly the same" (Diamond, 1983). Some authors suggest enrolling the help of other patients so that potential non-compliers can discuss the advantages and disadvantages of taking treatment.

\section{Supervision}

The value of supervision and, in particular, of intrusive follow-up when patients fail to attend, receives relatively little attention in the literature. Some patients make a rational decision as to whether or not they will take medication and hold to this decision consistently and in the face of whatever action the psychiatric team looking after them

Box 2. Involving the patient

Establish an maintain a joint approach to treatment

Listen to the patient

Empathise

Provide comprehensible information

Negotiate treatment

\section{Box 3. Supervision}

Be persistant

Intrude where necessary

Involve a multi-disciplinary team, friends and family

may take. Many others, however, are ambivalent. They may simply be undecided as to whether medication is of benefit, or they may change their view from one day to the next. This fluctuation may or may not be the result of residual symptoms of illness. Whether it is or not, it means that attempts to get the patient to agree to take treatment are much more likely to be successful if they are persistent (see Box 3). Supervision should involve both health staff and relatives or friends, and the reasons why it is being done need to be explained. Although some people find supervision intrusive, people are more likely to think of medication as important if they see that others think it is important (Diamond, 1983).

\section{The drug regime}

Complexity of prescribed treatment is associated with poor compliance, therefore drug regimes should be kept as simple as possible (see Box 4). Despite the perhaps surprisingly equivocal research findings described above, it seems inescapable that people who suffer significant side-effects will be more likely to stop taking the drug which causes them. Overt symptoms, such as tremor, are probably less important than how a drug makes the patient feel (Van Putten \& May, 1978). The difficulty, of course, is that few options are available beyond the two obvious ones of using the minimum dose of neuroleptic necessary to keep the patient well and treating Parkinsonian symptoms associated with neuroleptics. The new atypical antipsychotic drugs may change this. Finally, prescribing depot neuroleptics is associated with improved compliance and has the added benefit that staff can be sure whether or not someone is receiving the drug.

Box 4. The drug regime

Keep it simple

Attend to side-effects

Use depot medication if in doubt 
Box 5. Information and education

Give patients information about

Medication

Side-effects
Box 7. Work with family and relatives

Provide education and training

Suggest or intiate contact with self-help groups

Question whether home is the best place for the patient

research evidence on this point is more equivocal than might be expected). The lack of drive and negative symptoms associated with schizophrenia mean that any treatment programme which requires energetic participation is less likely to be successful. Patients who are confused or who have difficulty in remembering to take their medication can be helped by prompts of various kinds, such as leaving the tablets beside their toothbrush, as well as by making use of relatives and friends.

\section{Work with family and relatives}

Family members provide most of the care which people with chronic mental disorders receive and are the people best able to supervise the taking of their medication, always assuming that they are persuaded of its value (see Box 7). Education and training can improve relatives' understanding of psychosis and they can be trained in dispensing of medication. They can be put in touch with self-help and support groups and may in turn feel better able to encourage their relative to comply with treatment. In some cases the best family intervention may consist of encouraging the patient to leave home.

\section{Attending to the administrative and physical aspects of treatment}

Patients who have to wait a long time to see their doctor, or who wait at home for a community nurse who misses the appointment, are less likely to attend, or wait, for treatment next time (see Box

Box 8. Attending to the physical and administrative aspects of the treatment

Waiting times

Location

Time of day

Prompting 
8). Different people prefer different environments. Some find their fortnightly visit to the out-patient clinic enjoyable and see it as a chance to meet friends they have made through the hospital. Others may be reminded of unhappy events or be embarrassed to be seen there and prefer to receive their treatment at home or in their GP's surgery. Many people with schizophrenia are uncomfortable around other people, either as a result of anxiety or their morbid beliefs. Patients who work need special arrangements so that the amount of time they need to take off can be reduced to a minimum. The circumstances of follow-up need to be tailored to the preferences of the individual. As community treatment facilities are developed the 'one size fits all' approach to the out-patient treatment of people with schizophrenia becomes less acceptable than ever. The most common reason for non-compliance may be forgetfulness, magnified by the effect of symptoms on a patient's cognitive function (Falloon, 1984). Administrative strategies can be devised to address this. Both simplifying the drug regime and prompting will help. After that there is the familiar hierarchy of means of supervising the taking of medication ranging from out-patient attendance, through day hospital to having community nurses administer medication in patients' own homes.

\section{Conclusions}

Didactic education seems to be of little help. Despite some methodological problems, the research seems to show that talking to patients about their treatment more than we do routinely at least has an effect on what they say about taking medication. It seems unlikely that what patients say is completely unrelated to what they do and it is reasonable to assume that interventions such as those described here make a difference. How much of a difference remains to be seen. Finally, there is a value to supervision and to intrusive follow-up, which has not been demonstrated in randomised trials but which emerges from the literature and accords, I think, with clinical experience.

\section{References}

Baekeland, F. \& Lundwall, L. (1975) Dropping out of treatment: a critical review. Psychological Bulletin, 82, 738-783.

Boczkowski, J., Zeichner, A. \& DeSanto, N. (1985) Neuroleptic compliance among chronic schizophrenic out-patients: an intervention outcome report. Journal of Consultingand Clinical Psychology, 53, 666-671.

Brown, C., Wright, R. \& Christensen, D. (1987) Association between type of medication instruction and patients' knowledge, side effects and compliance. Hospital and Community Psychiatry, 38, 55-60.

Buchanan, A. (1992) A two-year prospective study of treatment compliance in patients with schizophrenia. Psychological Medicine, 22, 787-797.

Corrigan. P., Liberman, R. \& Engel, J. (1990) From non compliance to collaboration in the treatemt of schizophrenia. Hospital and Community Psychiatry, 41, 1203-1211.

Diamond, R. (1983) Enhancing medication use in schizophrenic patients. Journal of Clinical Psychiatry, 44, 7-14.

Eckman, T., Wirshing, W., Marder, S., et al (1992) Technique for training schizophrenic patients in illness self-management: a controlled trial. American Journal of Psychiatry, 149, 1549-1555.

Falloon, I. (1984) Developing and maintaining adherence to longterm drug-taking regimens. Schizophrenia Bulletin, 10, 412-417.

Hogan T., Awad, A. \& Eastwood, R. (1983) A self-report scale predictive of drug compliance in schizophrenics: reliability and descriminative validity. Psychological Medicine, 13, 177-183.

Kemp, R., Hayward, P., Applewhaite, G., et al (1996) Compliance therapy in psychotic patients: randomised controlled trial. British Medical Journal, 312, 345-349.

Ley, P. \& Morris, L. (1984) Psychological aspects of written information for patients. In Contributions to Medical Psychology, Vol. 3 (ed. S. Rachman), pp. 117-149. Oxford: Pergamon Press.

MacPherson, R., Jerrom, B. \& Hughes, A. (1996) A controlled study of education about drug treatment in schizophrenia. British Journal of Psychiatry, 168, 709-717.

Miller, W. \& Rollnick, S. (1991) Motivational Interviewing: Preparing People to Change Addictive Behaviour. New York: Guilford Press.

Overall, J. E. \& Gorham, D. R. (1962) The brief psychiatric rating scale. Psychological Reports, 10, 799-812.

Soskis, D. \& Bowers, M. (1969) The schizophrenic experience. Journal of Nervous and Mental Disease, 149, 443-449.

Streicker, S., Amdur, M. \& Dincin, J. (1986) Educating patients about psychiatric medications: failure to enhance compliance. Psychosocial Rehabilitation Journal, 9, 15-28.

Van Putten, T. \& May, P. (1978) Subjective response as a predictor of outcome in pharmacotherapy: the consumer has a point. Archives of General Psychiatry, 35, 477-480.

\section{Multiple choice questions}

1. Important predictors of compliance include:

a socio-demographic factors

b optimism regarding the future

c knowledge of illness

d the characteristic of the therapist

e illness severity.

2. Features of a patient's drug regime which have been linked to poor compliance include:

a the number of drugs being taken

b the number of times per day drugs are taken

c the use of atypical antipsychotic drugs

$\mathrm{d}$ the use of oral, rather than depot medication

e collection of drugs from GP as opposed to hospital. 
3. Techniques that usually improve compliance include:

a increasing the level of supervision

b attending to drug side-effects

c negotiating the dose with the patient

d providing the patient with information about drugs

e seeing family members regularly.

\begin{tabular}{|cllllll|}
\hline MCQ answers & & & & & \\
1 & & 2 & & 3 & \\
a & F & a & T & a & T \\
b & T & b & T & b & T \\
c & F & c & F & c & T \\
d & T & d & T & d & F \\
e & F & e & F & e & T \\
\hline
\end{tabular}

\section{Commentary}

\section{Jenny Fisher}

I have enjoyed reading Buchanan's paper very much. It is clear, thoughtful and contains much valuable information. As I read it, I began to think it would make a good foundation for a short factual leaflet for carers. However, they are unlikely to read it in its present form, since many would find the scientific style and wording hard going. Words such as compliance, drugs and intrusive have connotations and need to be used with care by professionals. The National Schizophrenia Fellowship uses the term adherence instead of compliance.

Buchanan covers one of the main anxieties which carers face, what to do when their patient decides to stop taking medication. I found it comforting to read, since much of it confirms many of the things I have discovered by trial and error over the years.

It is good to read that professionals now largely accept that neuroleptics are valuable and that failure to take medication leads to relapse, which can in turn, lead to risk for the individual or others. This is not a new concept to carers who have had to cope with the problems over the years. However, information about how they might help to prevent this happening is very welcome.

Carers are recruited at random from the general public, they are not chosen. They come with all the inhibitions, ignorance, prejudice and fear which the public holds for mental illness. The first thing many new carers ask is for information. Some immediately set about obtaining this with a vengeance while others are just totally baffled and give up. Information is best given individually and psychiatric staff are best placed to do this. Carers need the relevant information as soon as possible, as they often have other responsibilities, such as families and jobs, to attend to in additon to caring for their patient.

The paper points clearly to the connection between individual care provided by interested professionals and good adherence to treatment. Tender loving care encourages a sense of well-being and raises self-esteem. Carers might also benefit from this kind of interest.

Buchanan recognises that family members provide most of the care which people with chronic mental illness receive and are best placed to supervise the taking of medication by the patient. It is common for carers to monitor medication, but for this to be effective they also need information about the illness: what is likely to happen; what kind of medication is needed and its action on the patient; side-effects and risks. They also need to know that it is not the end of the world if a tablet is missed, and to be a party to decisions to increase or decrease the dose or change the timing of medication. Some of the suggestions in this paper

Jenny Fisher trained as a registered general nurse and has been a carer for the past 17 years. She worked in nursing middle management until two years ago. One of her children has schizophrenia and Asperger Syndrome. Her intrests include all aspects of serious mental illness. She is a trustee for the National Schizophrenia Fellowship (28 Castle Street, Kingston upon Thames, Surrey, KT1 1SS) and is an Honorary Parliamentary Officer. 